\title{
ESTUDIO GEOLÓGICO PRELIMINAR DEL ALERO ROCOSO CERRO CASTILLO (PATAGONIA AUSTRAL, CHILE) ${ }^{1}$
}

PASCAL BERTRÁN"

\section{RESUMEN}

El estudio del relleno sedimentario del alero de Cerro Castillo (Patagonia meridional) dejó en evidencia cuatro fases principales: 1) fase antigua compuesta de sedimentos detríticos ricos en carbonatos procedentes de la degradación de la pared, 2) el desarrollo de un suelo marrón, actualmente descarbonatado hacia $4.800{ }^{14} \mathrm{C} \mathrm{AP}$, sugiriendo una cobertura de bosque alrededor del sitio durante un período húmedo, 3) la formación de un humus coluvial, aún carbonatado, asociada al desarrollo de la estepa, y 4) recientes depósitos ricos en estiércol de ovejas. El estudio de los microartefactos (líticas, carbones y fragmentos óseos) contenidos en los depósitos muestra fluctuaciones importantes en la estratigrafía. Estas fluctuaciones se asignan a varios factores, de origen antrópico (sucesión de ocupaciones humanas) o naturales (redistribución de los vestigios por el escurrimiento, disolución de los restos óseos en el suelo fósil).

PALABRAS CLAVES: Alero rocoso, suelos fósiles, tafonomía, Holoceno, Patagonia austral.

\section{PRELIMINARY GEOLOGICAL STUDY OF CERRO CASTILLO ROCKSHELTER (PATAGONIA, SOUTHERNMOST CHILE)}

\begin{abstract}
The study of the Cerro Castillo rockshelter (Patagonia, southernmost Chile) identify four main sedimentary phases: 1) deposition of calcareous detritus derived from the fragmentation of the rock wall during the early Holocene, 2) development of a decalcified forest brown soil dated to ca. $4800{ }^{14} \mathrm{C} \mathrm{BP}$, probably in connection with a wet period, 3) formation of a colluvial calcareous humus horizon, associated with a steppic vegetation and 4) deposition of cattle excrements. The microartefact content (lithic industry, charcoal, bone fragments) of the layers shows significant fluctuations along the stratigraphy. These are attributed either to anthropogenic (multiple human occupation) or natural factors (artefact redistribution due to overland flow, bone dissolution in the palaeosoil).
\end{abstract}

KEYWORDS: Rockshelter, palaeosoils, taphonomy, Holocene, southernmost Chile.

1 Versión francesa en Legoupil y Bertran 2006 "L'abri Pedro Cardenas et l'abri Cerro Castillo", Rapport de la Mission Archéologique de Patagonie, Ministère des Affaires Etrangères (France), multigraphié, 41p.

INRAP/ PACEA, UMR 5199 du CNRS, Bâtiment de Géologie, Avenue des facultés, Bordeaux, F-33405. pascal.bertran@inrap.fr 


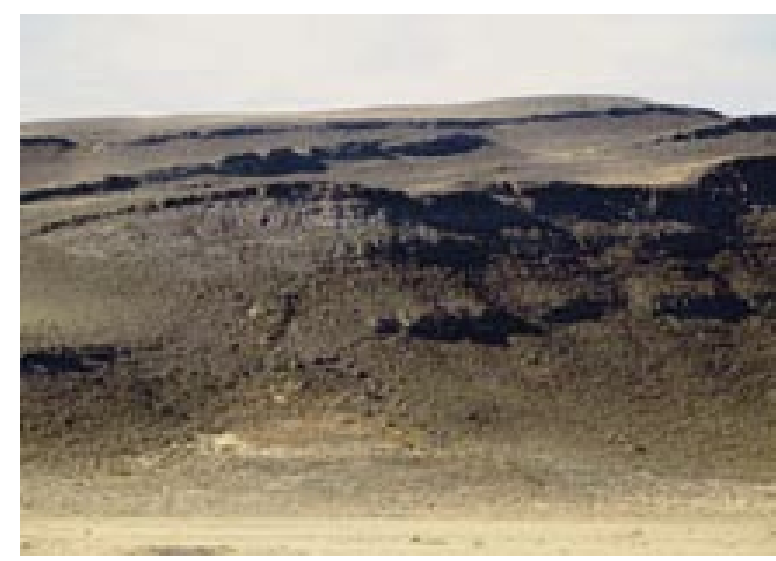

Fig. 1. Vista general de la vertiente y del alero. Éste se sitúa al centro de la fotografía, en la segunda barra rocosa.

\section{CUADRO GEOMORFOLÓGICO}

El alero rocoso Cerro Castillo 1 se localiza en una formación de conglomerados de areniscas y pelitas de origen marino, asignados entre el Cretáceo superior y el Oligoceno, y que afloran a nivel regional en una amplia banda paralela al eje de la

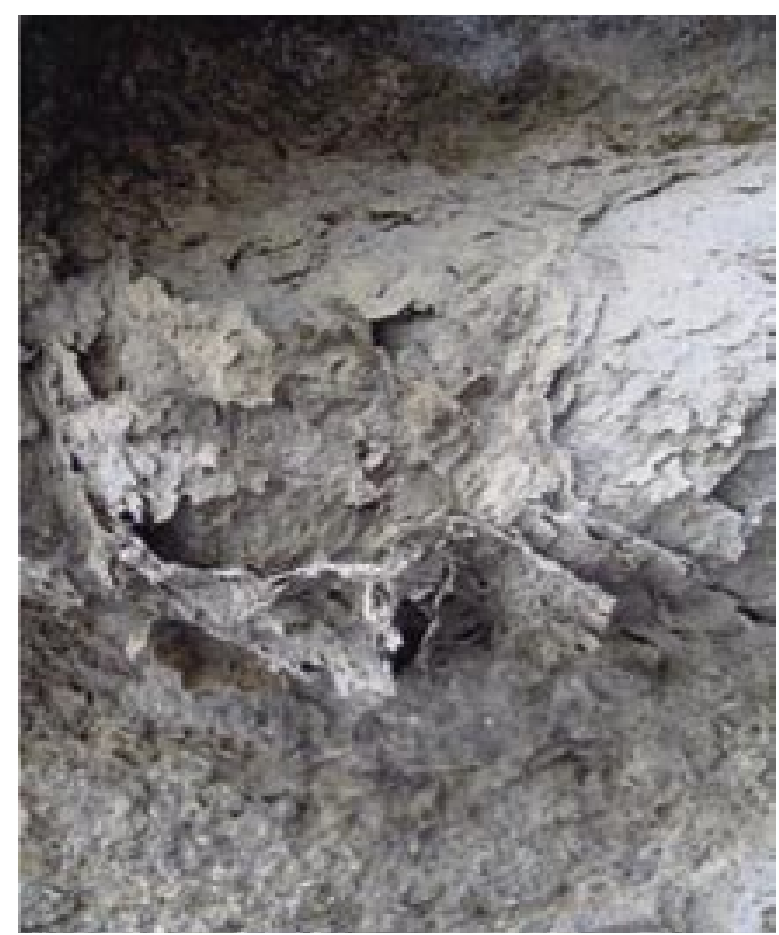

Fig. 3. Exfoliación de la pared arenosa. La alteración de la pared resulta en un sedimento arenoso carbonatado.

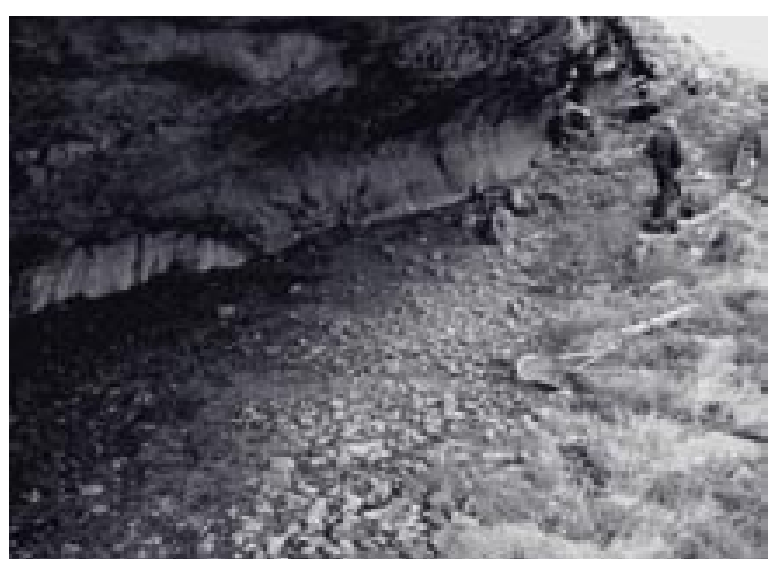

Fig. 2. Vista del alero antes de la excavación. Se observa la disminución de la granulometría del material detrítico desde el talud externo hacia el fondo del alero.

Cordillera andina (Mapa Geológico, ENAP, 1978). En el sector estudiado, la formación presenta una pendiente de cerca de $20^{\circ}$ hacia el este y los bancos más resistentes determinan una serie de pequeñas barras rocosas escalonadas sobre el lado de los valles. El alero se cavó bajo una de estas barras (Fig. 1). Sus dimensiones son de aproximadamente 5 metros de fondo y una treintena de metros de longitud, alcanzando una altura de 3 metros en la entrada. De manera bastante típica, se observa un talud con materiales gruesos, de bloques métrico, en la entrada del sector (Fig. 2). Este talud es abastecido a la vez por la fragmentación de la pared expuesta y por contribuciones laterales procedentes de conos detríticos que entran lateralmente en el alero. La parte interna del talud presenta una textura más fina, de origen mixto: desagregación de la piedra del techo del alero (Fig. 3), aportes coluviales abastecidos por los conos detríticos laterales, aportes eólicos y acumulación de excrementos de ovejas. Este relleno presenta una pendiente paralela a la pared de cerca de $5^{\circ}$.

El sitio domina un antiguo valle glaciar pleistoceno que pertenece al sistema de Lago Toro, en el macizo del Paine (Fig. 4).

Según Marden y Clapperton (1995) y Glasser et al. (2008), el conjunto de morrenas frontales que bordean el actual Lago del Toro corresponde al último máximo glacial (LGM, 23-25 ka). Las morrenas externas, que no han sido aún fechadas, deben por ello corresponder a la penúltima glaciación o, considerando los datos obtenidos por Kaplan et al. 


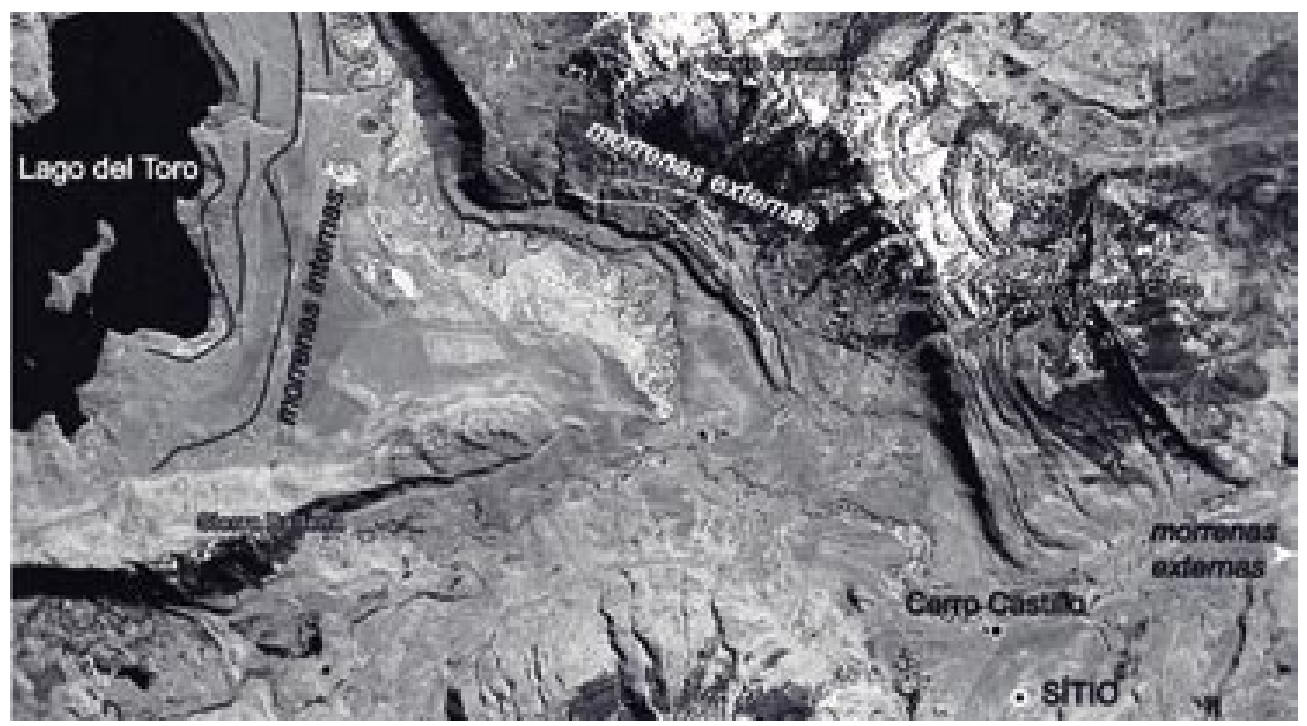

Fig. 4. Localización del sitio.

(2005) en el Lago Buenos Aires incluso a una fase antigua del Pleistoceno. Ello pone de manifiesto que el sitio, y la porción de valle situado al pie del mismo quedaron liberados de hielo ya durante el Pleistoceno superior, sin perjuicio de su cercanía al frente glacial para el LGM.

Los registros climáticos de los últimos años, bastante incompletos, efectuados en Cerro Castillo (511'00' S, 72²1'00 W) muestran que el clima se caracteriza por una temperatura media anual aproximadamente de 5 a $6^{\circ} \mathrm{C}$ y precipitaciones escasas, de 250 milímetros para el año 2005 a 460 milímetros para el año 1990, según los datos del Departamento de Hidrología de Santiago y de Mauricio Álvarez estanciero en Cerro Castillo (Fig. 5). Caídas de nieve se producen de junio a octubre. Estos valores sitúan el sitio cerca del límite entre dos grandes unidades fitogeográficas características de Patagonia, la estepa con pastizales de Festuca y Stipa de las regiones semiáridas $(\mathrm{P}<400 \mathrm{~mm}$, Moore 1978) y el bosque caducifolio con Nothofagus pumilio y antarctica de las regiones más lluviosas, más próxima al Océano Pacífico (400 mm $<\mathrm{P}<800 \mathrm{~mm}$, ibid.).

Los objetivos de este trabajo son de dos órdenes: 1) describir la estratigrafía del alero para proponer una primera interpretación de la dinámica sedimentaria y pedológica, y

2) aportar algunos elementos de valoración sobre las condiciones de preservación en los niveles arqueológicos.

\section{DATOS Y MÉTODOS}

La estratigrafía del alero se estableció a partir de los perfiles expuestos por los sondeos arqueológicos. Se tomaron distintas muestras, desde los cortes, para análisis del contenido de carbono orgánico y de carbonatos por pirolisis y espectrometría infrarroja, con ayuda de un analizador Leco CS-125. Por otra parte, el contenido de microartefactos (líticos, carbones y fragmentos de hueso) separados por tamizado seco se cuantificó, en una columna de sedimentos de 50 x $50 \mathrm{~cm}$. Los cálculos fueron efectuados por niveles de $5 \mathrm{~cm}$ de espesor.

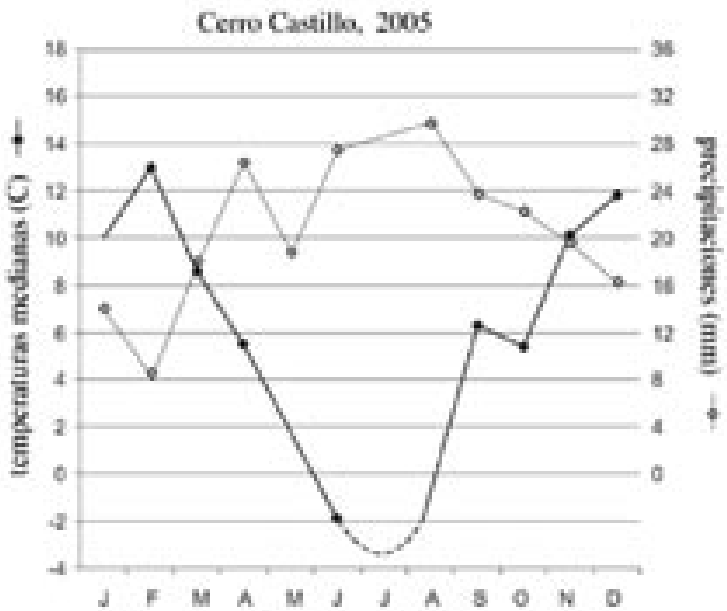

Fig. 5. Datos climáticos (fuente Dpto. Hidrología, Santiago). 


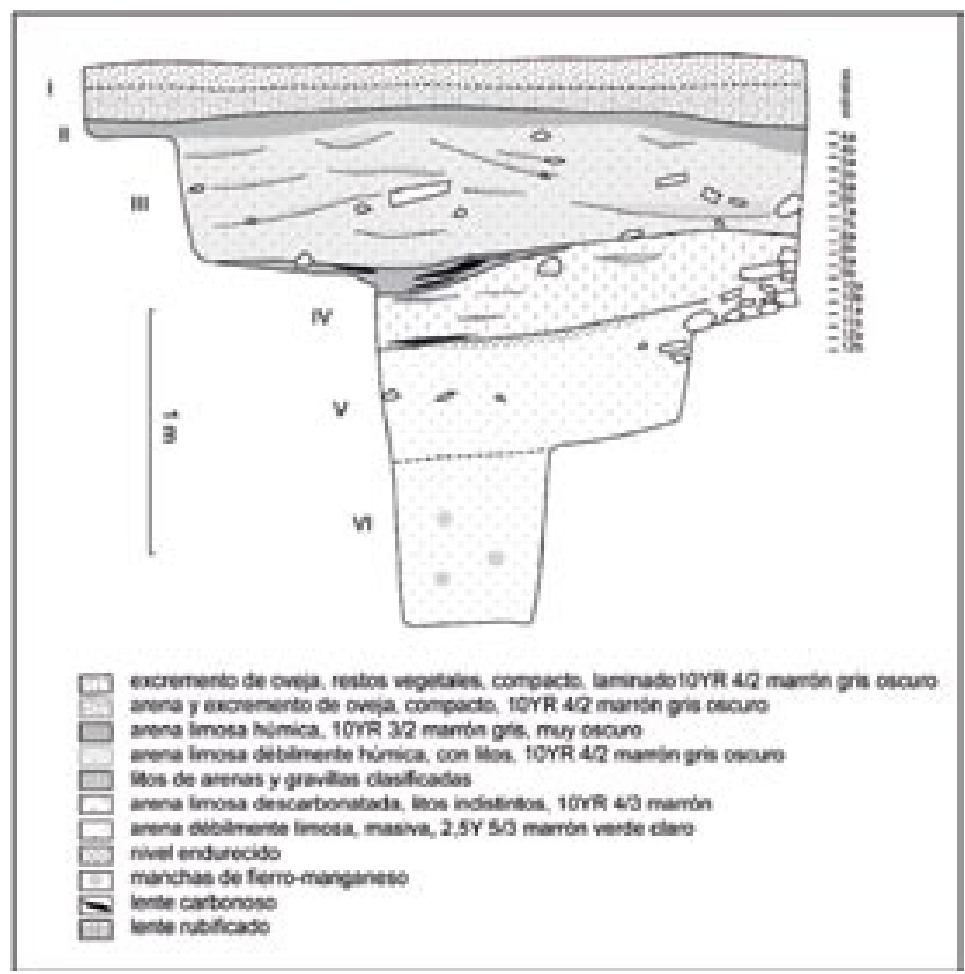

Fig. 6. Esquema estratigráfico, perfil longitudinal Sur.

\section{ESTRATIGRAFÍA}

Se observaron unidades sedimentarias (US) bastante homogéneas en toda el área excavada. Incluyen, de arriba hacia abajo (Fig. 6, 7):

I) Unidad rica en excremento de ovejas mezcladas con arenas limosas calcáreas procedentes de la degradación del techo del alero. Estos niveles, de una veintena de centímetros de espesor, son de color marrón gris oscuro, estructuralmente son masivos o presentan, localmente, una estructura laminar relacionada con la compactación resultante del pisoteo de ganado.

II) Unidad de humus areno-limoso pulverulento, rica en raíces y en pequeños restos vegetales mal decompuestos, de color marrón gris muy oscuro. Este nivel, de 5 a 10 centímetros de espesor, se interpreta como el horizonte de humus (A11) del suelo del alero previo a su uso por el ganado. Las observaciones efectuadas en otros aleros vecinos, menos frecuentados por las ovejas, muestran la presencia de una cubierta vegetal discontinua, herbácea y arbustiva. Actualmente no subsiste ningún rastro de ésta en el sitio (Fig. 8). Las gravas están cubier- tas por puntuaciones blancas, que probablemente corresponden a carbonatos secundarios.

III) Unidad areno-limosa de humus, marrón gris oscuro, con estradas entrecruzadas ${ }_{\lambda}$ más o menos

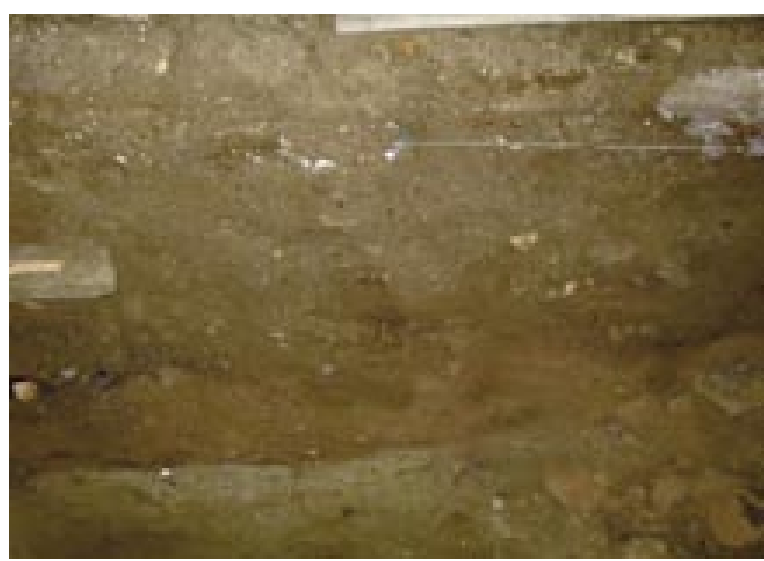

Fig. 7 Perfil longitudinal del sondeo. Se distingue en la base, niveles detríticos marrón amarillo (capas V y VI), luego el horizonte $\mathrm{B}$ de color marrón de un suelo fósil (capa IV), y un horizonte orgánico A coluvial grueso (capas II y III). La parte superior de la estratigrafía (sobre la lienza) está constituida por excremento de oveja mezclado con sedimento arenoso resultantes de la desagregación de la pared (capa I). 


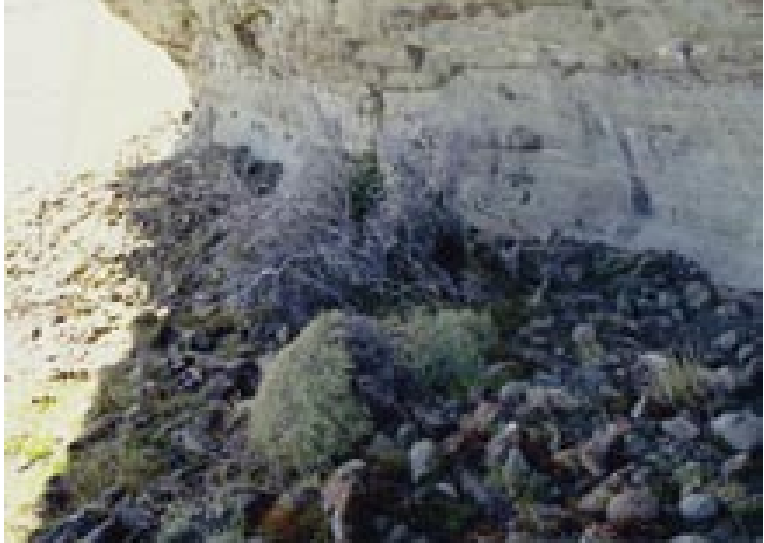

Fig. 8. Vegetación que coloniza un alero poco frecuentado por las ovejas. Esta foto representa, probablemente, una buena idea del estado del sitio antes de la importación de las ovejas en la región.

marcadas (Fig. 9). La estratificación se caracteriza por niveles de gravas o de arenas bien clasificadas, de extensión decimétrica a métrica en corte longitudinal (perpendicular a la pared), que corresponden a pavimentos y acumulaciones arenosas asociadas a escurrimientos de agua. Este nivel, cuyo espesor alcanza 30 a 50 centímetros, se interpreta como un horizonte A coluvial.

IV) Unidad areno-limosa marrón, es un estrato indiferenciado de una treintena de centímetros de espesor. Al contrario que en las unidades anteriores, la matriz se presente descarbonatada. Este nivel corresponde al horizonte $\mathrm{B}$ de alteración de un paleosuelo marrón descarbonatado desarrollado sobre coluviones. El límite inferior, brutal, muestra el frente de descarbonatación del suelo.

V) Unidad de arena calcárea con poco limo, masivo, de color marrón a marrón-verde claro. La parte superior (5 a 10 centímetros de espesor), está localmente cementada en particular, en la parte anterior del alero. La induración está vinculada a la reprecipitación de los carbonatos procedentes del horizonte $\mathrm{B}$ subyacente. Estos materiales corresponden a colusiones. La ausencia de una estratificación puede relacionarse con la acción de procesos secundarios sobre los depósitos, en particular, los ciclos de hielo/deshielo.

VI) La base de la secuencia está constituida por arenas marrón grises, con motas ferro-manganésicas sobre las gravas. Éstas atestiguan la presencia de

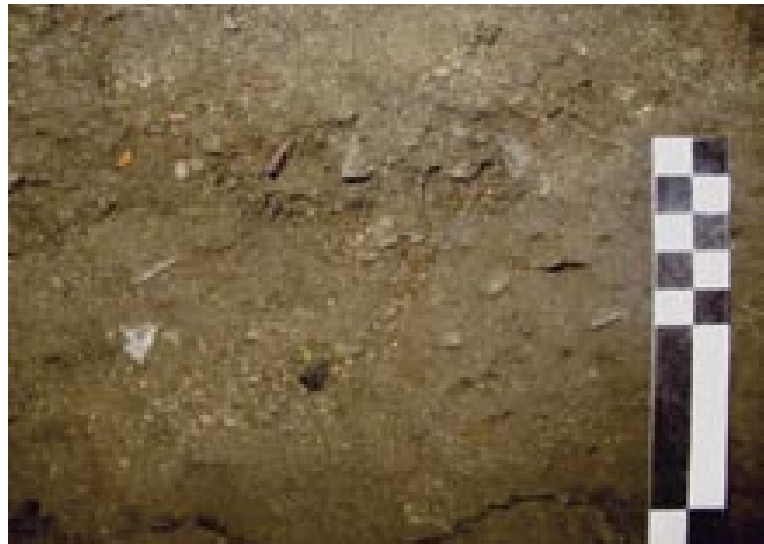

Fig. 9. Vista de detalle de las capas 1 a 3. Se distinguen, arriba, la capa de excremento de ovejas, luego un nivel de humus, rico en raíces. Este nivel se desarrolla sobre un material areno-limoso con lentes de pequeñas gravillas que corresponden al fondo de regueros cavados por escurrimiento.

fluctuaciones en el nivel capa de agua asociadas a la proximidad del substrato rocoso.

\section{INTERPRETACIÓN}

El conjunto de los depósitos observados corresponde a coluviones bastante homogéneos cuyo origen es probablemente complejo (desagregación del techo del alero, contribuciones eólicas y de materiales fluidos abastecidos por los taludes que penetran lateralmente en el alero). Por ello la mayoría de los criterios en los cuales se basa la estratigrafía propuesta son de carácter pedológico. Permiten distinguir cuatro grandes unidades:

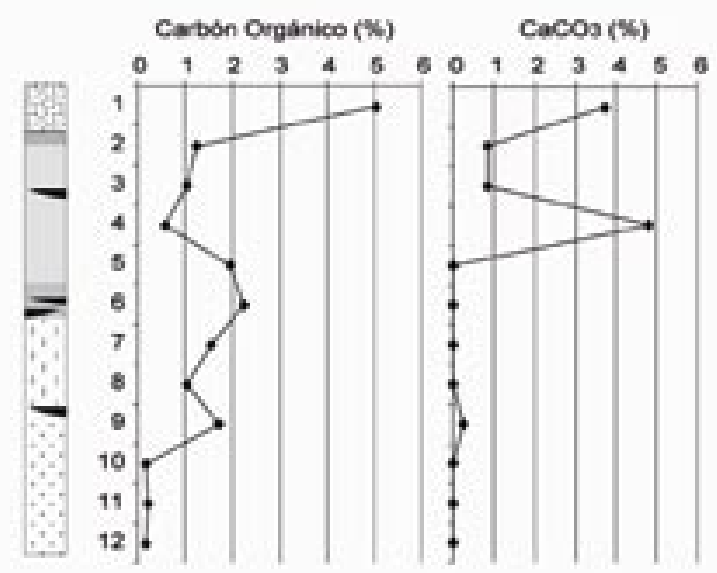

Fig. 10. Medidas del carbón orgánico y del calcáreo (CaCO3). 
a) En la base de la secuencia, las unidades $V$ y VI, están carbonatadas y poco teñidas por materia orgánica (Fig. 10). Estas características sugieren que los coluviones se fueron estableciendo en un medio ambiente escasamente vegetado que era poco propicio para la alteración. La fecha $14 \mathrm{C}$ obtenido en el fogón 2 (San Roman y Morello 2003), que cubre estas capas, indica que su deposición es anterior a aproximadamente 4.800 AP. Coherente con esta interpretación, los estudios palinológicos sobre la historia regional de la vegetación (Heusser 1987, Villa-Martínez y Moreno 2007, Moreno et al. 2009), sugieren que el desarrollo postglacial del bosque de Nothofagus es tardío en el sur de la Patagonia y Tierra de Fuego: en diversos lugares, la estepa con manchas de bosque de Nothofagus típica del comienzos del Holoceno persiste hasta 5 a 7 ka AP.

b) La unidad IV, descarbonatada, corresponde al horizonte $\mathrm{B}$ de un Paleosuelo. El desarrollo del paleosuelo y la alteración consecutiva de los depósitos sugieren la presencia de una cubierta

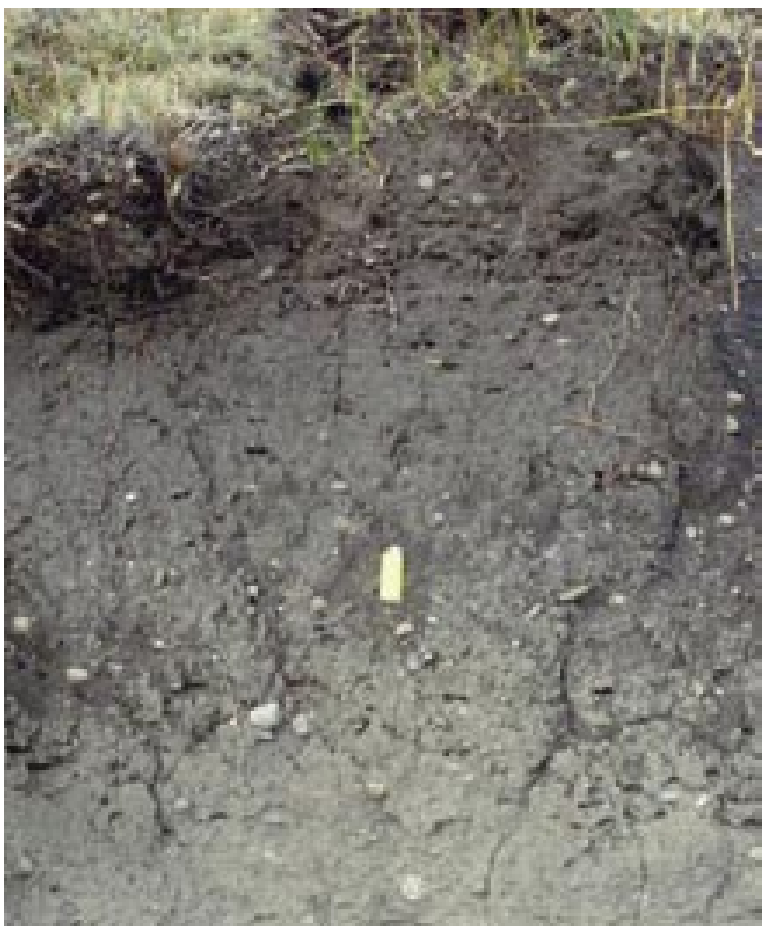

Fig. 11. Suelo iso-húmico bajo la vegetación esteparia, sector de Cerro Castillo. Este suelo se caracteriza por un horizonte Ah marrón gris grueso desarrollado sobre materiales morénicos. vegetal densa y una disminución de la velocidad de sedimentación en el alero. La presencia de un horizonte $\mathrm{B}$ marrón (brunifié) contrasta con los suelos actualmente observados en los alrededores del sitio, que corresponden a suelos iso-húmicos típicos de estepa y se caracteriza por un horizonte de humus Ah, grueso, desarrollado sobre la roca-madre poco alterada (horizonte C) (Fig. 11).

Sin embargo se observaron algunos suelos marrones comparables (véase los calcisols de la clasificación francesa de suelos Baize y Girard 1995) sobre materiales morénicos cercanos a Río Rubens, aproximadamente $50 \mathrm{~km}$ al sur de Puerto Natales, bajo el bosque de Nothofagus pumilio $(400 \mathrm{~mm}<$ P > 800 mm, Moore, 1978) (Fig. 12). La formación del paleosuelo, por ende, podría corresponder a un período caracterizado por el desarrollo de bosque caduco sobre el sitio.

Coherente con esta hipótesis, los datos polínicos obtenidos por Moreno et al. (2009) en Lago Guanaco, situado a una cincuentena de kilómetros al noroeste de Cerro Castillo,

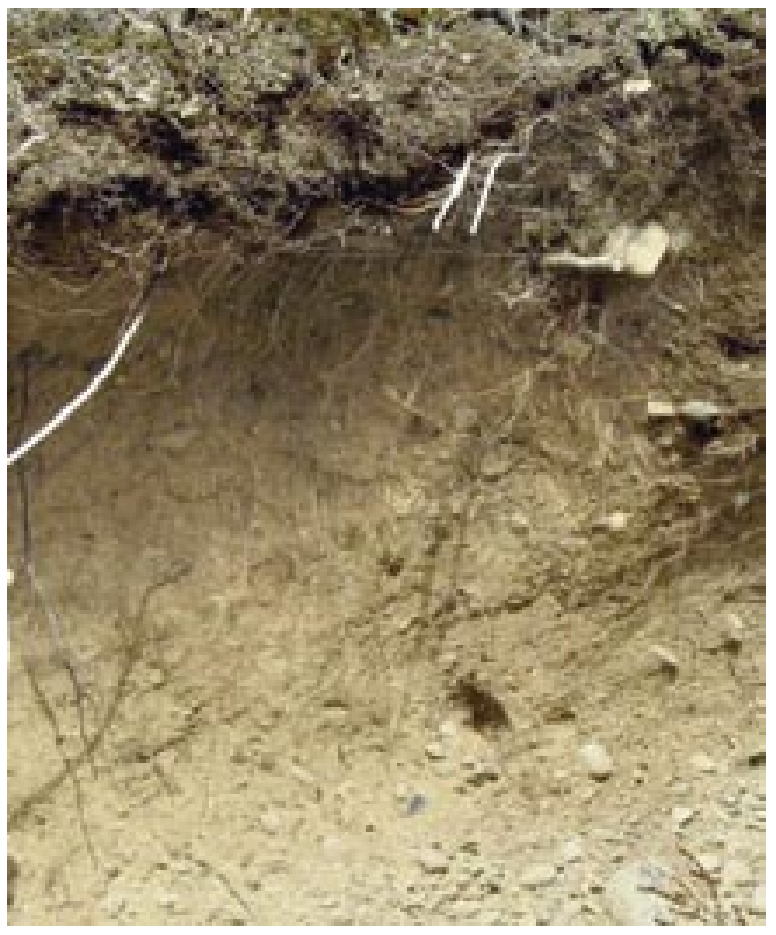

Fig. 12. Suelo marrón (calcisol) bajo un bosque de hayas recientemente vaciado, sector de Río Rubens. Este suelo se caracteriza por un humus poco grueso y un horizonte $\mathrm{B}$ descarbonatado de color marrón, desarrollado sobre materiales morénicos. 
muestran un pico en el desarrollo del bosque de Nothofagus entre 4200 y 4700 AP, seguido por un largo período durante el cual la estepa domina el paisaje. No se observan valores comparables en la relación Nothofagus / Poacées sino durante los últimos 500 años, es decir, durante la Pequeña Edad Glacial.

c) Las unidades II y III, carbonatadas y ricas en materia orgánica, sugieren, la presencia de vegetación en los alrededores del sitio que, descompuesta es progresivamente incorporada en el depósito por la actividad biológica y los procesos coluviales. En comparación con la capa subyacente, la alteración y la descarbonatación de los sedimentos son escasas, ya sea porque la velocidad de sedimentación aumentó (posible resultado de la antropización del sitio), o debido a una disminución de las precipitaciones. Este último factor puede ser más importante ya que acompaña la instalación de la estepa actualmente presente en el sector, escenario que es coherente con datos polínicos de numerosas secuencias regionales. Estos últimos sugieren una apertura de los bosques y un aumento de los taxones esteparios después de 3 ka AP, (Heusser 1987, Moore 1978, Moreno et al. 2009).

d) La unidad I, en gran parte compuesto de excrementos de oveja, indica el desarrollo de la ganadería ovina en la región, resultado de la colonización de la Patagonia por poblaciones europeas.

\section{ARQUEOESTRATIGRAFÍA Y DATOS TAFONÓMICOS: CONTRIBUCIÓN DE LA GRANULOMETRÍA DEL MATERIAL LÍTICO}

La excavación suministró abundante material arqueológico, la mayor parte del cual se concentra en las unidades sedimentarias II, III y IV. Para establecer una secuencia arqueoestratigráfica lo más detallada posible, en una zona test (esquina noroeste de F15), los objetos de más de $2 \mathrm{~cm}$ de longitud fueron levantados con registro en tres dimensiones (ver Legoupil 2009 , este volumen). En paralelo el material más pequeño se recogió con un tamiz de 2 milímetros, siguiendo niveles artificiales de $5 \mathrm{~cm}$ de espesor. Los resultados obtenidos indican significativas variaciones verticales de abundancia (Fig. 13).

Se observan cuatro picos de abundancia bastante desiguales del material lítico (A1 a A4). El más importante (A3) corresponde a la base de la unidad III, es decir, el fogón 1 de San Roman y Morello (2003). El análisis de granulometría del material lítico pone de manifiesto que, en estos niveles, la frecuencia es similar a la observada en conjuntos procedentes de eventos de talla experimental. Esta composición es la esperada para niveles de ocupación que no han tenido selección por los procesos sedimentarios que participaron en su enterramiento (Schick 1986; Lenoble 2003; Bertran et al. 2005). Estos niveles están separados por lentes, a la vez, menos ricos

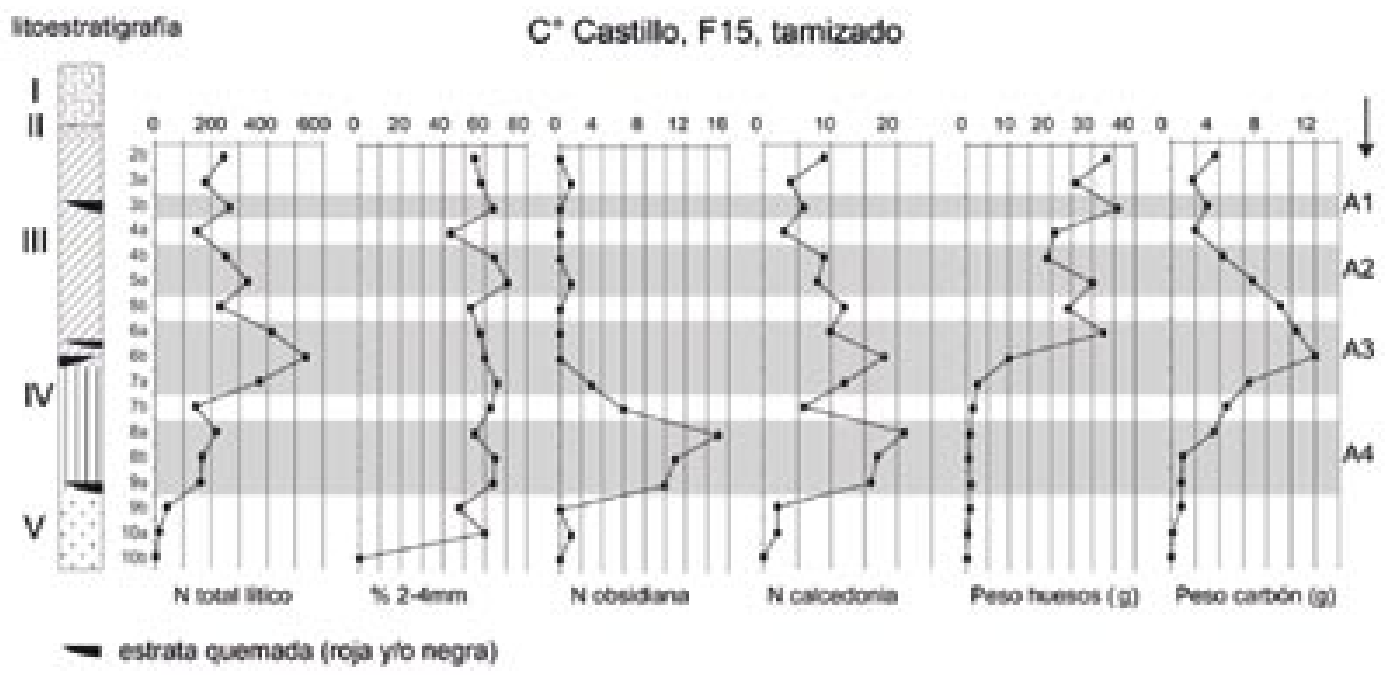

Fig.13. Distribución vertical de los distintos tipos de vestigios arqueológicos recogidos en el tamizado, cuadrado F15, y esquema de la lito estratigrafía. 
en objetos y con escasos elementos pequeños. La composición granulométrica del material lítico, en estos pequeños lentes intermediarios, se aleja ostensiblemente de aquella observada en conjuntos experimentales y sugiere una selección granulometríca. Las observaciones de terreno sugieren que el escurrimiento puede ser la causa de la selección. Sobre el diagrama CD propuesto por Lenoble (2003), establecido a partir de experimentos con la acción de escurrimientos, los puntos representativos de estos niveles sugieren la superficie de concentraciones residuales de objetos, es decir, conjuntos próximos a su lugar de origen pero empobrecidos por los escurrimientos (Fig. 14).

La frecuencia de otros elementos encontrados en el tamizado también establece información interesante. Así el número de esquirlas de obsidiana

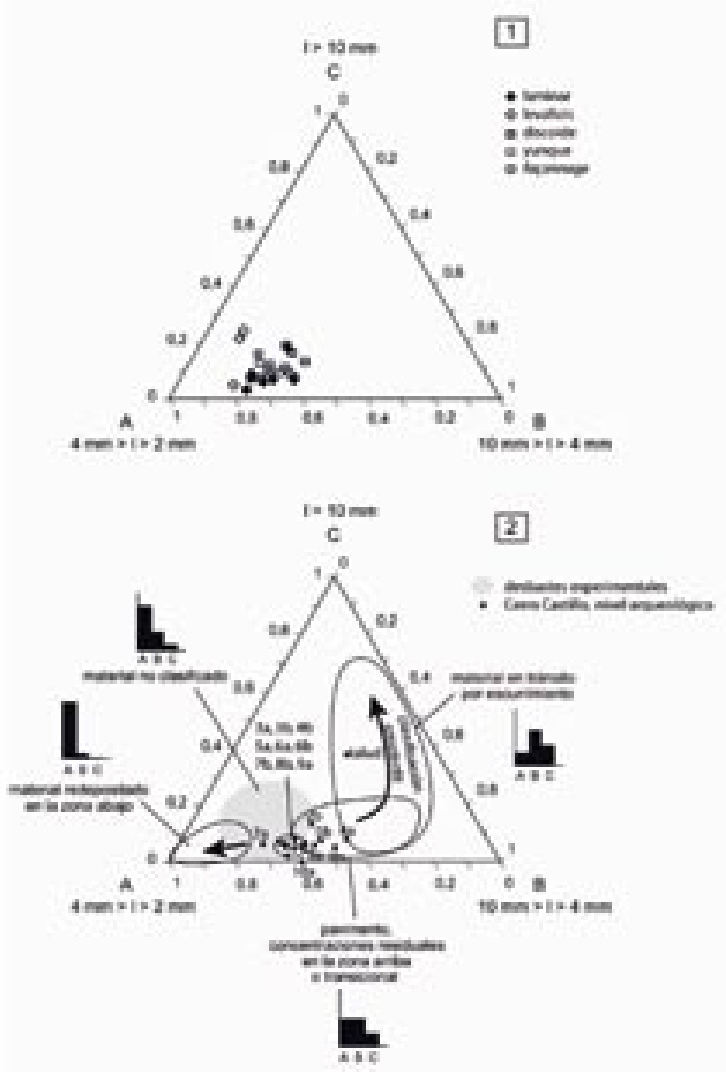

Fig. 14. Composición de granulometría de los vestigios líticos. 1: composición de los desbastes experimentales, según Bertrán et al., no publicado; 2: composición de los niveles arqueológicos de Cerro Castillo y superficies de las composiciones que resultan de una selección granulométrica por escurrimiento, según Lenoble (2003). y calcedonia permite claramente definir el nivel arqueológico A4, en la base de la secuencia. En efecto, estas materias primas están muy bien representadas en este nivel en comparación con su escaso o casi ausencia en otros.

La cantidad de restos óseos presenta variaciones muy importantes a lo largo de desarrollo de la estratigrafía. La parte inferior de la secuencia prácticamente carece de huesos, mientras que éstos son muy abundantes en las capas superiores. Esta ausencia no se vincula probablemente a factores de origen antrópico sino debe asignarse a una alteración que acompaña el del paleosuelo. En la parte superior, los picos de abundancia de huesos siguen, aproximadamente, los del material lítico. Eso tiende a indicar que el factor principal de acumulación de los vestigios óseos sobre el sitio es de origen antrópico. Contribuciones sustanciales hechas por carnívoros y aves rapaces no deben, sin embargo, ser excluidas, como lo prueba la elevada cantidad de restos (oveja, guanaco...) actualmente presentes en superficie (Fig. 15).

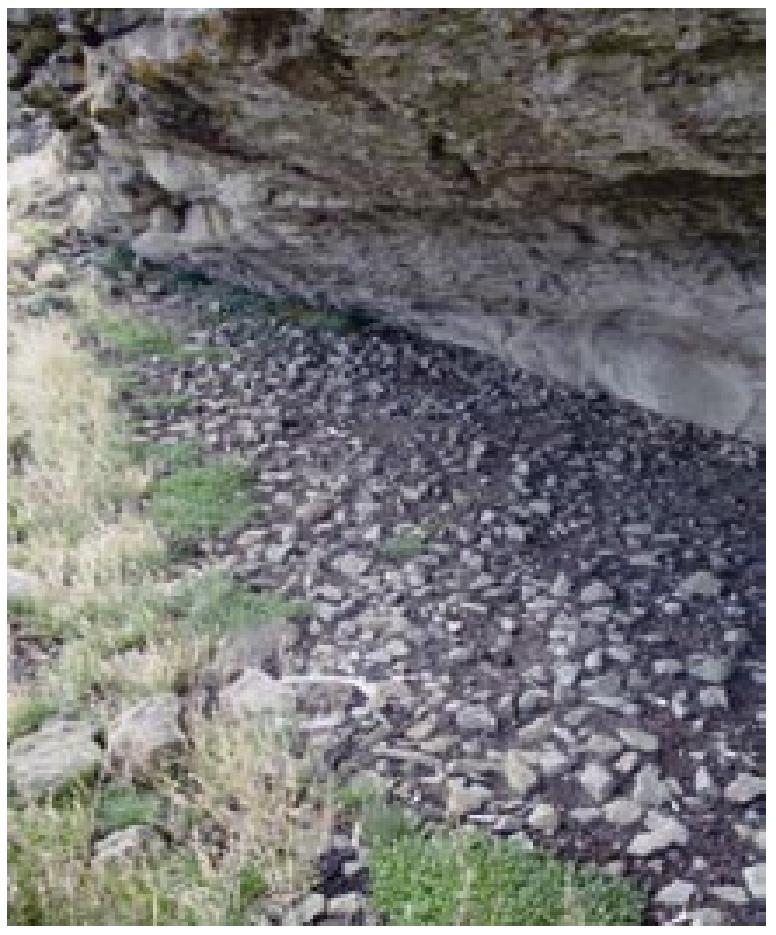

Fig.15. Osamentas de ovejas y guanacos dispersados sobre la sperficie del alero. Las contribuciones de carnívoros y aves rapaces constituyen, probablemente, una parte no desdeñable del material acumulado en el relleno del abrigo. 

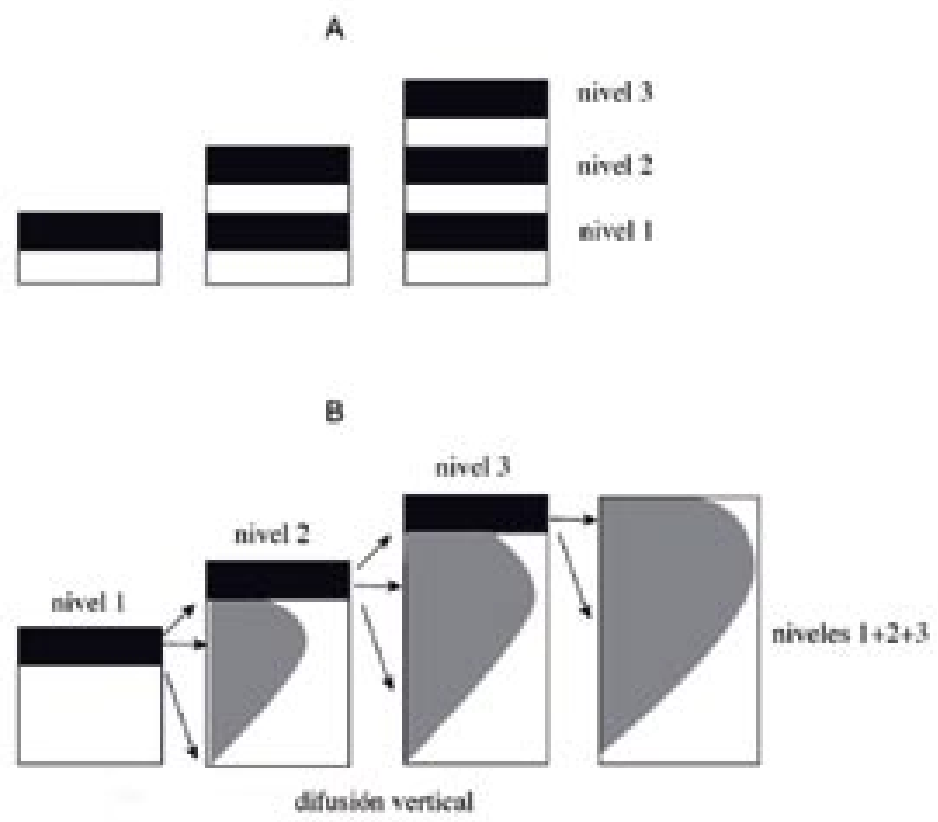

Fig. 16. Distribución de los objetos arqueológicos en un contexto donde la sedimentación se acompaña de una difusión vertical del material (B, modificado según Siriainen, 1977), o no (A). La distribución B corresponde a lo que se observa en Cerro

Castillo para los carbones; para el material lítico y óseo, la distribución es intermedia entra A y B. En el caso de Cerro Castillo, la difusión vertical se acompaña probablemente de una redistribución lateral vinculada al escurrimiento en la pendiente.

Contrariamente a los otros elementos arqueológicos, la cantidad de carbones de madera recogidos en el tamizado fino no presenta fluctuaciones estratigráficas marcadas. Se observa en único pico de abundancia, en el nivel arqueológico A3. Este tipo de distribución sugiere que la acumulación de los carbones no resulta estrictamente de los mismos fenómenos que el resto del material. El esquema propuesto por Siriainen (1977) permite dar cuenta de este tipo de distribución (Fig. 16). Implica una mobilidad vertical significativa de los carbones, que borra los cambios bruscos perceptibles para los otros tipos de vestigios. Este proceso de mobilidad puede explicarse por el carácter más móvil de los carbones en relación a objetos más densos como los huesos líticos especialmente cuando están expuestos a la acción del viento y del escurrimiento.

En conclusión, el estudio del material de esta columna pone de relieve cuatro niveles arqueológicos principales. Estos niveles estuvieron expuestos durante más o menos tiempo a la acción de los procesos naturales, en particular, el viento, el escurrimiento y la alteración; y su fisonomía resulta, a la vez, de procesos antrópicos y fenómenos naturales.
Varios índices, que se refieren tanto al material lítico como a carbones de madera ponen de manifiesto que una parte del material arqueológico sufrió una redistribución más o menos importante, en particular un efecto residual por el escurrimiento. En tal contexto, es probable que la distribución espacial de los niveles de ocupación fueron perturbados, al menos localmente (cf. Lenoble 2003). Sin embargo, sin una excavación sobre una gran superficie la magnitud de estas perturbaciones sigue siendo difícil de estimar. El estudio del estado de superficie de los huesos podría ofrecer información útil desde un punto de vista tafonómico. Una observación preliminar realizada por F. Martin indica que este estado varía significativamente según los niveles e implica un tiempo variable de exposición a los procesos naturales de la superficie del suelo. Ello sugiere en consecuencia, una degradación variable en los distintos niveles. En este sentido, el material procedente de los estratos artificiales 2 y 3 (cf. Fig. 6) está muy fragmentado y erosionado (Fig. 17). La conservación es mejor en los estratos 4 y 5 donde sólo se alteraron algunos huesos (fisura, exfoliación, abrasión) mientras que otros tienen un aspecto fres- 


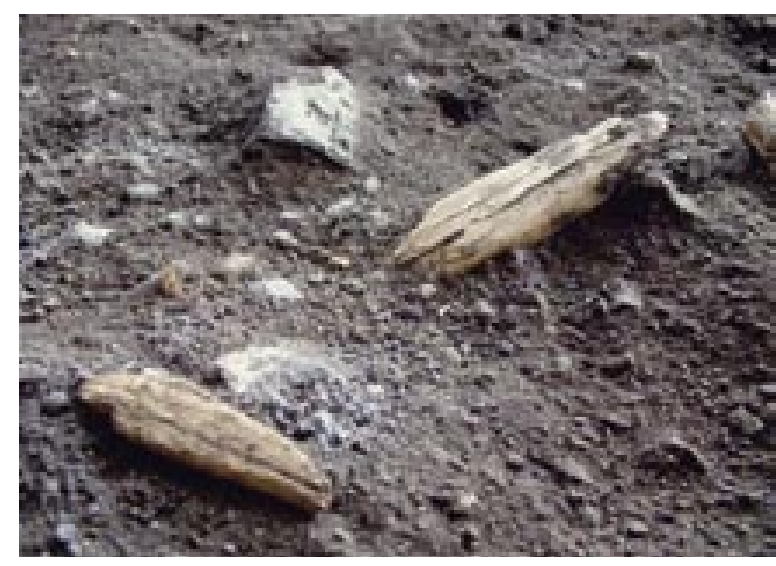

Fig.17. Fisuración y exfoliación de los fragmentos de huesos en la parte superior de la unidad III.

co, a veces con huellas de corte muy visibles (Fig. 18). Por fin, en los estratos 6 a 9, algunos huesos preservados parecen muy desmineralizados pero no presentan fisuras importantes.

\section{AGRADECIMIENTOS}

El presente estudio fue financiado por la DGCID (Direction de la Coopération Internationale et du Développement) del Ministerio de Relaciones Exteriores de Francia.

Tradución: D. Legoupil y F. Morello.

\section{BIBLIOGRAFÍA}

BAIZE D. y GIRARD M. C., 1995 - Référentiel pédologique. INRA, Paris, $332 \mathrm{p}$.

BERTRAN P., LENOBLE A., LACRAMPE F., BRENET M., CRETIN C. y MILOR F., 2005 - Le site aurignacien de Combemenue à Brignac-la-Plaine (Corrèze) : apport de la géoarchéologie et de l'étude de l'industrie lithique à la compréhension des processus taphonomiques. Paleo, 17, p. 7-29.

GLASSER N.F., JANSSON K.N., HARRISON S. y KLEMAN J., 2008 - The glacial geomorphology and Pleistocene history of South America between $38^{\circ} \mathrm{S}$ and $56^{\circ} \mathrm{S}$. Quaternary Science Reviews, 27, pp. 365-390.

HEUSSER C. J., 1987 - Quaternary vegetation of southern South America. En INQUA International Congress, 12. Editado por A. A. Balkema, Rotterdam; Brookfield VT, Pays-Bas (Monographie) pp. 197-221^

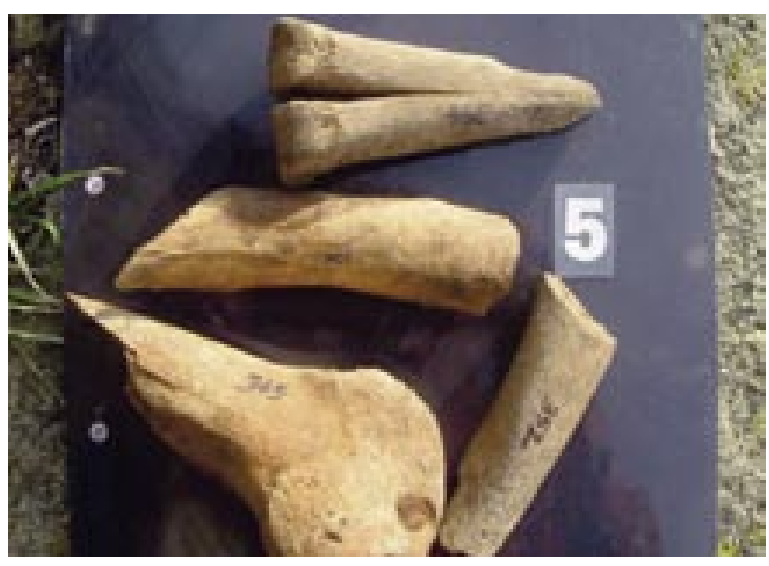

Fig.18. Huesos poco alterados en la parte intermedia de la unidad III.

LENOBLE A., 2003 - Le rôle du ruissellement dans la formation des sites préhistoriques : approche expérimentale. Thèse de doctorat, Université de Bordeaux 1, 306 p.

MAPA GEOLOGICO, XII Region Magallanes y Antartica chilena, Chile. Empresa nacional del Petroleo, dept. Exploraciones, esc. 1:500 000, 1978.

MARDEN C.J. y CLAPPERTON C.M., 1995 - Fluctuations of the Southern Patagonian Icefield during the last glaciation and the Holocene. Journal of Quaternary Science, 10, pp. 197-209.

MOORE D. M., 1978 - Post-glacial vegetation in the South Patagonian territory of the giant ground sloth, Mylodon. Botanical Journal of the Limnean Society of London, 77 (3), p. 177-202.

MORENO P.I., FRANÇOIS J.P., VILLA-MARTINEZ R.P. y MOY C.M., 2009 - Millenial-scale variability in Southern Hemisphere westerly wind activity over the last 5000 years in SW Patagonia. Quaternary Science Reviews, 28, pp. 25-38.

SIRIAINEN A., 1977 - Pieces in vertical movement - a model for rockshelter archaeology. Proceedings of the Prehistoric Society, 43, p. 349-353.

SCHICK K. D., 1986 Stone Age sites in the making. Experiments in the formation and transformation of archaeological occurrences. BAR International Series 319, 313 p.

VILLA-MARTINEZ R. Y MORENO P.I., 2007 - Pollen evidence for variations in the southern margin of the westerly winds in SW Patagonia over the last 12,600 years. Quaternary Research, 68, p. 400-409. 